\title{
Integrated network pharmacology and molecular docking approaches to reveal the synergistic mechanism of multiple components in Venenum Bufonis for ameliorating heart failure
}

\author{
Wei Ren ${ }^{1}$, Zhiqiang Luo ${ }^{2}$, Fulu Pan ${ }^{3}$, Jiali Liu ${ }^{1}$, Qin Sun ${ }^{1}$, Gang Luo ${ }^{1}$, Raoqiong Wang ${ }^{1}$, Haiyu Zhao ${ }^{4}$, Baolin \\ Bian $^{4}$, Xiao Xiao ${ }^{5}$, Qingrong Pu ${ }^{1}$, Sijin Yang ${ }^{\text {Corresp., }}{ }^{1}$, Guohua Yu ${ }^{\text {Corresp. } 2}$ \\ ${ }^{1}$ Affiliated Traditional Chinese Medicine Hospital, Southwest Medical University, Luzhou, China \\ 2 School of Life Sciences, Beijing University of Chinese Medicine, Beijing, China \\ ${ }^{3}$ School of Chinese Materia Medica, Beijing University of Chinese Medicine, Beijing, China \\ 4 Institute of Chinese Materia Medica, China Academy of Chinese Medical Sciences, Beijing, China \\ 5 Beijing National Laboratory for Molecular Sciences, Key Laboratory of Analytical Chemistry for Living Biosystems, CAS Research/Education Center for \\ Excellence in Molecular Sciences, Institute of Chemistry, Chinese Academy of Sciences, Beijing, China \\ Corresponding Authors: Sijin Yang, Guohua Yu \\ Email address: ysjimn@sina.com, 202002016@bucm.edu.cn
}

Venenum Bufonis (VB), also called Chan Su in China, has been extensively used as a traditional Chinese medicine (TCM) for treating heart failure (HF) since ancient time. However, the active components and the potential anti-HF mechanism of VB remain unclear. In the current study, the major absorbed components and metabolites of VB after oral administration in rats were first collected from literatures. A total of 17 prototypes and 25 metabolites were gathered. Next, a feasible network-based pharmacological approach was developed and employed to explore the therapeutic mechanism of VB on HF based on the collected constituents. In total, 158 main targets were screened out and considered as effective players in ameliorating HF. Then, the VB components-main HF putative targets-main pathways network was established, clarifying the underlying biological process of VB on HF. More importantly, the main hubs were found to be highly enriched in adrenergic signalling in cardio-myocytes. After verified by molecular docking studies, four key targets (ATP1A1, GNAS, MAPK1 and PRKCA) and three potential active leading compounds (bufotalin, cinobufaginol and 19-oxo-bufalin) were identified, which may play critical roles in cardiac muscle contraction. This study demonstrated that the integrated strategy based on network pharmacology and molecular docking was helpful to uncover the synergistic mechanism of multiple constituents in TCM. 


\section{Integrated network pharmacology and molecular docking approaches to}

\section{2 reveal the synergistic mechanism of multiple components in Venenum Bufonis}

\section{3 for ameliorating heart failure}

4 Wei Ren*1, Zhiqiang Luo*2, Fulu Pan*3, Jiali Liu ${ }^{1}$, Qin Sun ${ }^{1}$, Gang Luo ${ }^{1}$, Raoqiong Wang ${ }^{1}$,

5 Haiyu Zhao ${ }^{4}$, Baolin $\mathrm{Bian}^{4}$, Xiao Xiao ${ }^{5}$, Qingrong $\mathrm{Pu}^{1}$, Sijin Yang ${ }^{1}$ and Guohua $\mathrm{Yu}^{2}$

$6{ }^{1}$ Affiliated Traditional Chinese Medicine Hospital, Southwest Medical University, Luzhou

7 646000, China

$8{ }^{2}$ School of Life Sciences, Beijing University of Chinese Medicine, Beijing 102488, China

$9{ }^{3}$ School of Chinese Materia Medica, Beijing University of Chinese Medicine, Beijing 102488,

10 China

$11{ }^{4}$ Institute of Chinese Materia Medica, China Academy of Chinese Medical Sciences, Beijing

12 100700, China

$13{ }^{5}$ Beijing National Laboratory for Molecular Sciences, Key Laboratory of Analytical Chemistry

14 for Living Biosystems, CAS Research/Education Center for Excellence in Molecular Sciences,

15 Institute of Chemistry, Chinese Academy of Sciences, Beijing 100190, China

16 Corresponding Author:

17 Sijin Yang ${ }^{1}$, Guohua $\mathrm{Yu}^{2}$

$18{ }^{1}$ Affiliated Traditional Chinese Medicine Hospital, Southwest Medical University, No.182

19 Chunhui Road, Longmatan District, Luzhou 646000, Sichuan Province, China.

$20{ }^{2}$ School of Life Sciences, Beijing University of Chinese Medicine, the northeast corner of the

21 intersection of yangguang south street and baiyang east road, Fangshan District, Beijing 102488,

22 China.

23 E-mail addresses: ysjimn@sina.com (S. Yang); 202002016@bucm.edu.cn (G. Yu) 
*These authors contributed equally.

\section{Abstract}

Venenum Bufonis (VB), also called Chan Su in China, has been extensively used as a traditional Chinese medicine (TCM) for treating heart failure (HF) since ancient time. However, the active components and the potential anti-HF mechanism of VB remain unclear. In the current study, the major absorbed components and metabolites of VB after oral administration in rats were first collected from literatures. A total of 17 prototypes and 25 metabolites were gathered. Next, a feasible network-based pharmacological approach was developed and employed to explore the therapeutic mechanism of VB on HF based on the collected constituents. In total, 158 main targets were screened out and considered as effective players in ameliorating HF. Then, the VB components-main HF putative targets-main pathways network was established, clarifying the underlying biological process of VB on HF. More importantly, the main hubs were found to be highly enriched in adrenergic signalling in cardio-myocytes. After verified by molecular docking studies, four key targets (ATP1A1, GNAS, MAPK1 and PRKCA) and three potential active leading compounds (bufotalin, cinobufaginol and 19-oxo-bufalin) were identified, which may play critical roles in cardiac muscle contraction. This study demonstrated that the integrated strategy based on network pharmacology and molecular docking was helpful to uncover the synergistic mechanism of multiple constituents in TCM.

Keywords: Venenum Bufonis, heart failure, network pharmacology, molecular docking

\section{Introduction}

Heart failure (HF), a multifactorial degenerative disease, occurs when the heart is not able 
45

46

47

to pump blood efficiently to satisfy the oxygen and nutritional needs of the body (Asano et al. 2019). HF affects over 26 million people worldwide and continues to represent a major burden

for public health due to its high mortality, morbidity and healthcare expenses (Di Palo \& Barone 2020; Lother \& Hein 2016). The incidence rate of HF rises in magnitude with age and the major etiologies were coronary heart disease, abnormal heart valves and hypertension (Akkineni et al. 2019). Western medicines, such as angiotensin converting enzyme (ACE) inhibitors, diuretics, $\beta$ adrenergic blockers, angiotensin receptor I antagonists and positive inotropic agents, are currently the main treatment programs for HF (Xu et al. 2020; Yang et al. 2020). However, longterm use of these chemical drugs will lead to a series of adverse reactions like electrolyte depletion, fluid depletion, and hypotension (Jia et al. 2020). Therefore, novel alternative or synergetic anti-HF therapies are greatly needed.

Venenum Bufonis (VB) is the dried white secretion of the auricular and skin glands of Bufo bufo gargarizans Cantor or Bufo melanostictus Schneider (He et al. 2019; Yun et al. 2009). As a precious traditional Chinese medicine (TCM), VB has long been used for treating heart failure, arrhythmia, swells, sore throat, pains, cancers and many other diseases (Liang et al. 2008; Pan et al. 2020). Extensive natural product studies have indicated that VB contains a high level of bufadienolides and many other constituents like alkaloids, cyclic amides, sterols, polypeptides, proteins, polysaccharides, and organic acids (Wei et al. 2019). Modern pharmacological studies have confirmed that VB exhibited a variety of pharmacological effects, including cardiotonic, antinociceptive, anti-tumor, anesthetic, anti-inflammatory as well as antimicrobial properties (Wei et al. 2020). Importantly, it is evident that VB exerts a strong cardiac excitatory effect like 
that of digitalis, and the drug possesses many advantages such as no accumulation, quick-acting and diuretic action (Wei et al. 2019). According to the Chinese Pharmacopoeia (2015 edition), VB is contained in many TCM prescriptions for the treatment of HD, such as Jiuxin Pill and Shexiang Baoxin Pill (Chinese Pharmacopoeia Commission, 2015). Although well-practiced in clinical medicine, the holistic pharmacological mechanisms of VB on HF are largely unknown. As an emerging field of pharmacology, network pharmacology delivers a systematic and holistic understanding of drug action and disease complexity, which shares key ideas with the integrality and systematicness of TCM theory (Luo et al. 2020; Zhang et al. 2020). An increasing body of evidence suggests that network pharmacology is a powerful tool to illuminate the integration synergistic mechanism of action of TCM from the multi-dimensional perspective (Chen et al. 2019; Miao et al. 2019). For example, the bioactive candidates and underlying mechanisms of Cichorium glandulosum for ameliorating type 2 diabetes mellitus was successfully elucidated using "compound-target" network analysis (Qin et al. 2019). The action mechanism of Carthamus tinctorius L. on cardiovascular disease was also elaborated based on the "compound-protein/gene-disease" network (Yu et al. 2019). However, due to the limitation of this method, many previous researches usually collected TCM components from related TCM databases to establish the compound-target map. The candidate compounds might not be in line with the components actually delivered into the blood circulatory system, which unavoidably produced unreal results (Ding et al. 2019; Zhang et al. 2018).

In this study, first the in vivo ingredients of VB after oral administration in rats were taken from the literature. Second, the VB- and HF-associated targets were predicted. Third, network 
87

construction and pathway enrichment analysis were used to explore the active components and the potential targets relevant to the treatment of HF with VB. Finally, molecular docking was performed to confirm the specific interactions between VB and the candidate targets. The above study not only provides a comprehensive understanding about the molecular mechanism of VB acting on HF, but also offers a rapid and effective strategy for screening desired compounds from TCM. The flowchart was illustrated in Fig. 1.

\section{Materials and methods}

2.1. In vivo constituents of $\mathrm{VB}$

A total of 42 in vivo constituents of VB have been reported, including 17 prototypes and 25 metabolites (He et al. 2012; Liang et al. 2008; Miyashiro et al. 2008; Ning et al. 2010; Tao et al. 2017; Xia et al. 2010; Xin et al. 2016; Zhu et al. 2013). The molecular 2D files of these constituents were downloaded from the ChemSpider database (http://www.chemspider.com/) and were saved in mol format (step 1 in Fig. S1). The details were listed in Table S1.

2.2. The prediction of VB-related targets

The predicted proteins targeted by the in vivo constituents of VB were screened from MedChem Studio (MedChem Studio, 3.0; Simulations Plus, Inc, Lancaster, CA, USA, 2012).

This software could efficiently capture the FDA-approved drugs that have similar chemical structures to the components in TCM (Yu et al. 2016). We picked out VB compound-drug pairs with high confidence scores $(\geq 0.6)$ and considered the target proteins of the known drugs as the VB-related targets. Other parameters were set as the default values (step 1 in Fig. S1). 
107

108

109

110

111

112

113

114

115

116

117

118

119

120

121

122

123

124

125

126

127

2.3. Known therapeutic targets for HF

In this study, two databases were employed in acquiring pathological targets for HF, by use of "heart failure" as the query. One was the DrugBank database (http://www.drugbank.ca/, version 5.1.1), which could provide detailed information on the drug targets and their links with human diseases (Griesenauer et al. 2019). Only drug-target interactions for FDA-approved antiHF drugs and potential human protein targets associated with HF were selected for further analysis. The second platform was the Online Mendelian Inheritance in Man (OMIM) database (http://www.omim.org/, updated on May 4, 2018), a constantly updated database of human genetic diseases and genes (step 1 in Fig. S1) (Hamosh et al. 2005).

\subsection{Protein-protein interaction (PPI) data}

The PPI information related to the putative targets of VB constituents and known therapeutic targets for HF was harvested by use of STRING (Search Tool for the Retrieval of Interacting Genes/Proteins) database (http://string-db.org/). This database could provide a global perspective of proteins and their functional interactions and associations (Jensen et al. 2009).

Results were limited to "Homo sapiens" and protein interactions with a confidence score greater than or equal to 0.4 would be selected. Other parameters were set as the default values (step 2 in Fig. S1).

2.5. Network construction and analysis

In order to illustrate the interaction among the ingredients, targets and diseases, a "ingredient-target-disease" network was built by introducing the information of candidate compounds of VB, putative targets of VB and HF-associated targets into Cytoscape software 
128 (version 3.6.0, Boston, MA, USA). This software is an efficient open source bioinformatics tool

129 for visualizing and analysing the complex biological networks (Shannon et al. 2003). Three

130 important topological characteristics ("degree", "betweenness", and "closeness"), which have

131 been described in our previous publication (Yu et al. 2018), were calculated to assess the central

132 attribute of each hub node in the network by use of the Cytoscape plugin "Network Analyzer".

133 And "degree" > median degree centrality, "betweenness" > median betweenness centrality and

134 "closeness" > median closeness centrality were adopted as the screening criteria to acquire the

135 critical targets (Luo et al. 2020). Other parameters were set as the default values (step 2 in Fig.

$136 \mathrm{~S} 1)$.

137 2.6. Pathway enrichment analysis

138 Kyoto Encyclopedia of Genes and Genomes (KEGG) pathways analysis and Gene

139 Ontology (GO) enrichment analysis were undertaken to explore the potential functions of the

140 pivotal target proteins involved in the VB-mediated treatment of HF(Nguyen et al. 2019a,

141 Nguyen et al. 2019b), by use of the Database for Annotation, Visualization and Integrated

142 Discovery (DAVID) system (http://david.abcc.ncifcrf.gov/home.jsp/, v6.8) (Dennis et al. 2003).

143 Relevant pathways with the false discovery rate (FDR)-corrected $P$-value $<0.05$ were considered

144 statistically significant. Other parameters were set as the default values (step 3 in Fig. S1).

2.7. Molecular docking simulation

146 Molecular docking studies were conducted to further verify the reliability of the potential targets using CDOCKER module implemented in Discovery Studio 2016 (DS 2016).

148 CDOCKER is a semi-flexible molecular docking analysis method based on the CHARMm force 
149 field, which can produce high-precision docking results, and it provides information on the

150 interaction binding energy and ligand-receptor docking mode. The three-dimensional (3D)

151 structures of the candidate compounds were generated using Chem3D Pro 12.0 and the

152 crystallographic structures of the proteins encoded by the candidate target genes were obtained

153 from the PDB database (http://www.rcsb.org/pdb/home/home.do), which was then decorated by

154 removing the ligands, adding hydrogen, removing water, optimizing and patching amino acids.

155 The binding site was defined by the ligand atoms, and the radius range was automatically generated. After each compound was docked, the 10 best conformations were obtained (Yang, 2020). Finally, CDOCKER interaction energies (CIEs) were used to assess the binding affinities between the core targets and the corresponding compounds. And the conformation corresponding to the lowest CIE was selected as the most probable binding conformation. All parameters used in calculation were default except for explained (step 4 in Fig. S1).

\section{Results and discussion}

\subsection{Putative targets for VB}

As shown in Table S2, a total of 260 potential targets of the in vivo components of VB were obtained by MedChem Studio. The results showed that the candidate compounds could act on multiple targets, and one target could also be linked to multiple components.

3.2. Known therapeutic targets for $\mathrm{HF}$

We collected 109 and 199 HF-related targets from DrugBank database and OMIM database, respectively. We then checked the data and eliminate redundant entries, leaving a final dataset of 
170

171

172

173

174

175

176

177

178

179

180

181

182

183

184

185

186

187

188

189

190

related targets, including NR1I2, MT-CO1, CA2, NR3C1, SHBG, ATP1A1, CYP17A1, PGR, NR3C2, MME, AR, ACE, PRKCA, CYP11B2, RXRA, PPARG, PIK3CG, PPARD, PDE4B, KCNMA1, MED1 and HIF1A.

3.3. Network and pathway analysis

To facilitate scientific interpretation of the complex relationships between VB and HF, a "chemical target-disease associated gene" network, comprising the VB-related targets and the HF-related targets, was constructed based on the PPI data from STRING database. As listed in Table S4, this network was composed of 461 nodes and 5009 edges. After computing the values of the topological features of all hubs, 158 major hubs were identified because they satisfied the criteria (degree cutoff $=19$, betweenness cutoff $=0.001659$, and closeness cutoff $=0.381426$ ) Among them, 93 hubs were VB-related targets, 65 hubs were HF-related targets. These major hubs may play a critical role in the entire interaction network. The specific information about the major hubs was shown in Table S5.

3.4. Potential mechanisms of VB in treating HF

To elucidate the biological process (BP), molecular function (MF), cellular components (CC) of these major hubs involved in, GO enrichment analysis was conducted on the major hubs. As shown in Fig. 2a-c, the top 10 significant GO entries $(P<0.05)$ were selected on the basis of $P$ value.

To deeply determine the function and systemic association of the main hubs, KEGG pathway enrichment analysis were conducted. The results indicated that these hubs were enriched in 101 significant pathways $(P<0.05)$. As shown in Fig. 2 d, the top 11 pathways were 
considered as the main biological processes involved in the treatment. To further identify the functional mechanisms of VB on HF, the candidate compounds-main VB-related targets-main pathways network diagram was generated and elucidated in Fig. 3. The hubs can be mainly divided into the following three functional modules (circles): signal transduction (including adrenergic signalling in cardio-myocytes, calcium signalling pathway, cAMP signalling pathway, dilated cardiomyopathy, long-term potentiation and cGMP-PKG signalling pathway), cardiovascular system (including cardiac muscle contraction, vascular smooth muscle contraction and hypertrophic cardiomyopathy), and neural regulation (including dopaminergic synapse and circadian entrainment). Interestingly, adrenergic signalling in cardio-myocytes was highly enriched in KEGG pathway analysis, which played a critical role in the regulation of cardiac muscle contraction (the top-ranked GO: Biological Process terms), suggesting that VB may impart therapeutic effects on HF majorly through adrenergic signalling in cardio-myocytes. As shown in Table S6, the VB putative targets associated with adrenergic signalling in cardio-myocytes include guanine nucleotide-binding protein $\mathrm{G}(\mathrm{s})$ subunit alpha (GNAS), adenylate cyclase 2 (ADCY2), ADCY5, protein phosphatase 1 catalytic subunit gamma (PPP1CC), protein phosphatase 2 catalytic subunit alpha (PPP2CA), protein phosphatase 2 catalytic subunit beta (PPP2CB), calcium voltage-gated channel subunit alpha1 C (CACNA1C), CACNA1D, ATPase $\mathrm{Na}^{+} / \mathrm{K}^{+}$transporting subunit alpha 1 (ATP1A1), mitogen-activated protein kinase 1 (MAPK1), protein kinase $\mathrm{C}$ alpha (PKCa, encoded by PRKCA). Fig. 4 depicts a graphical overview of adrenergic signalling in cardio-myocytes influenced by main putative targets of VB components. 
212 3.5. Molecular docking

213 Table 1 displayed the CIEs (top 5 for each target) of VB hit constituents against the active

214 sites of the screened targets in adrenergic signalling, including ATP1A1, GNAS, ADCY2,

215 ADCY5, PPP1CC, PPP2CA, PPP2CB, CACNA1C, CACNA1D, MAPK1 and PRKCA. The

216 results indicated that the VB-related components had been docked successfully with ATP1A1,

217 GNAS, MAPK1 and PRKCA, which may be the key targets involved in VB for the treatment of

218 HF. Herein, we selected four representative pairs of binding interactions to illustrate how the

219 four targets bound to their corresponding components (Fig. 5). The interplay between ATP1A1

220 and bufotalin was depicted in Fig. 5a and Fig. 5b. The hydroxyl groups on bufotalin could form

221 three hydrogen bonds with SER209, ARG191 and VAL712. Another key residue which involved

222 in interaction was MET157. The binding mode of GNAS and cinobufaginol was depicted in Fig.

223 5c and Fig. 5d. The hydroxyl groups could bind with LEU171, MET255, ASN254 and LYS300

224 by forming hydrogen bonds. The carbonyl on the lactone ring could also form hydrogen bond

225 with LYS305. In addition, the lactone ring could bind with GLU164 and LYS305 via pi-anion

226 and pi-cation interactions. Other interactions including alkyl and pi-alkyl were connected with

227 ALA303, TYR163 and LEU296. The action mode of MAPK1 and cinobufaginol was depicted in

228 Fig. 5e and Fig. 5f. Cinobufaginol could form five hydrogen bonds with LYS112, LYS52 and

229 TYR34. In addition, the lactone ring could bind with ASP109 via pi-anion interaction. Other

230 interactions including alkyl and pi-alkyl were connected with VAL37. The interplay between

231 PRKCA and 19-oxo-bufalin was depicted in Fig. 5g and Fig. 5h. The hydroxyl groups of 19-oxo-

232 bufalin could form two hydrogen bonds with PRO202 and LYS230. Another key residue which

Peer] reviewing PDF | (2020:05:49255:2:0:NEW 8 Sep 2020) 
233

234

235

236

237

238

239

240

241

242

243

244

245

246

involved in interaction was LEU200.

\section{Discussion}

Many pathways are involved in adrenergic signalling for the regulation of cardiac

contractile function. Among them, the best described is the mechanism mediated by $\beta$-adrenergic

receptor ( $\beta$-AR)-Gs-adenylate cyclase (AC) pathway (Baker 2014). Activation of $\beta$-AR-Gs-AC

plays an important role in increasing heart rate and force of myocardial contraction (Chen et al.

2020; Santulli \& Iaccarino 2016). According to our predicted results, VB could regulate $\beta$-AR-

Gs-AC pathway by targeting Gs (GNAS) and AC (ADCY2 and ADCY5). The regulation of

$\mathrm{Ca}^{2+}$ homeostasis is also important for the cardio-myocyte excitation and cardiac electrical

activity (Arakelyan et al. 2007). According to our predicted results, VB could regulate cardiac

$\mathrm{Ca}^{2+}$ cycling by targeting PP1 (PPP1CC), PP2A (PPP2CA, PPP2CB), and LTCC (CACNA1C,

CACNA1D). $\mathrm{Na}^{+} / \mathrm{K}^{+}$-ATPase, a ubiquitous membrane protein composed of two subunits

denoted as $\alpha$ and $\beta$, is also a critical regulator in maintaining the balance of $\mathrm{Ca}^{2+}$ in cardio-

myocytes (Orlov et al. 2020; Šeflová et al. 2017). An increasing body of evidence suggests that

bufadienolides in VB possess inhibition effects on $\mathrm{Na}^{+} / \mathrm{K}^{+}$-ATPase (Orlov et al. 2020; Sousa et

al. 2017), such as bufalin (Lan et al. 2018; Laursen et al. 2015), cinobufagin (Wang et al. 2014),

marinobufagenin (Strauss et al. 2019), arenobufagin (Cruz Jdos \& Matsuda 1993), and

hellebrigenin (Moreno et al. 2013). Therefore, the cardiotonic effect of VB may be mainly

through the suppression of $\mathrm{Na}^{+} / \mathrm{K}^{+}$-ATPase. Inhibition of the $\mathrm{Ca}^{2+} / \mathrm{PKC} \alpha / \mathrm{ERK} 1 / 2$ signal pathway

plays a significant role in attenuating the progression of heart failure (Braz et al. 2004; Molkentin

\& Robbins 2009). Several components from VB have been reported to inhibit the activity PKC $\alpha$

Peer] reviewing PDF | (2020:05:49255:2:0:NEW 8 Sep 2020) 
254 and ERK, such as bufalin (Wu et al. 2015), marinobufagin (Bagrov et al. 2000; Fedorova et al.

255 2003) and cinobufagin (Baek et al. 2015).

256 Based on the data analysis above, the bufadienolides may be the main active components in

257 VB, which exert anti-HF effects via synergistically acting on multiple targets in multiple

pathways. Among them, the positive inotropic effect of VB produced through the inhibition of

$\mathrm{Na}^{+} / \mathrm{K}^{+}$-ATPase has been demonstrated in many basic researches and clinical practices. The

molecular docking results indicated that the representative compounds could connect with the

active-site residues via various noncovalent interactions, including the hydrogen bonding, pi-

alkyl, pi-anion and pi-cation, etc, which was valuable for understanding of the action

mechanisms of VB. In addition, according to -CIE values, bufotalin, cinobufaginol and 19-oxo-

bufalin showed the best performance and thus were considered as the potential active leading

compounds of the corresponding targets. Further researches on other potential targets or

pathways are required to validate the predicted results.

\section{Conclusion}

In summary, the active components of VB and their synergistic mechanisms for alleviating

HF were successfully unveiled by network pharmacology coupled with molecular docking

approach. The adrenergic signaling involved in cardiac muscle contraction process was found to

be mainly responsible for the anti-HF effect of VB in silico. Four core targets and their

corresponding leading compounds were identified, which may provide valuable information for

further experimental validations and drug discovery. 


\section{Additional information and declarations}

\section{Acknowledgements}

276 This work was supported by the National Traditional Chinese Medicine Clinical Research Base 277 (Grant NO. [2020]33).

\section{Competing interests}

279 The authors declare that there are no competing interests.

\section{Author contributions}

281 Wei Ren performed the experiments, wrote the paper, approved the final manuscript.

282 Zhiqiang Luo performed the experiments, wrote the paper, approved the final manuscript.

283 Fulu Pan performed the experiments and wrote the paper, approved the final manuscript.

284 Jiali Liu analyzed the data, approved the final manuscript.

285 Qin Sun analyzed the data, approved the final manuscript.

286 Gang Luo prepared figures and/or tables, approved the final manuscript.

287 Raoqiong Wang prepared figures and/or tables, approved the final manuscript.

288 Haiyu Zhao provide software, approved the final manuscript.

289 Baolin Bian provide software, approved the final manuscript.

290 Xiao Xiao reviewed drafts of the manuscript, approved the final manuscript.

291 Qingrong Pu reviewed drafts of the manuscript, approved the final manuscript.

292 Sijin Yang conceived and designed the experiments, approved the final manuscript.

293 Guohua Yu conceived and designed the experiments, approved the final manuscript. 
294

295

296

297

298

299

300

301

302

303

304

305

306

307

308

309

310

311

312

313

314

\section{Data availability}

The following information was supplied regarding data availability:

The raw measurements are available in the Supplemental Files.

\section{Supplemental information}

Supplemental information for this article can be found online at http://

References

Akkineni S, Mohammed O, Pathiraj J, Devasia T, Chandrababu R, and Kunhikatta V. 2019. Readmissions and clinical outcomes in heart failure patients: A retrospective study. Clinical Epidemiology and Global Health. https://doi.org/10.1016/j.cegh.2019.11.002

Arakelyan KP, Sahakyan YA, Hayrapetyan LR, Khudaverdyan DN, Ingelman-Sundberg M, Mkrtchian S, and Ter-Markosyan AS. 2007. Calcium-regulating peptide hormones and blood electrolytic balance in chronic heart failure. Regulatory Peptides 142(3):95100. https://doi.org/10.1016/j.regpep.2007.02.001

Asano R, Abshire M, Dennison-Himmelfarb C, and Davidson PM. 2019. Barriers and facilitators to a 'good death' in heart failure: An integrative review. Collegian 26(6):651665. https://doi.org/10.1016/j.colegn.2019.09.010

Baek SH, Kim C, Lee JH, Nam D, Lee J, Lee SG, Chung WS, Jang HJ, Kim SH, and Ahn KS. 2015. Cinobufagin exerts anti-proliferative and pro-apoptotic effects through the modulation ROS-mediated MAPKs signaling pathway. Immunopharmacology and Immunotoxicology 37(3):265-273. DOI 10.3109/08923973.2015.1027916

Bagrov AY, Dmitrieva RI, Dorofeeva NA, Fedorova OV, Lopatin DA, Lakatta EG, and 
315

316

Droy-Lefaix MT. 2000. Cicletanine reverses vasoconstriction induced by the endogenous sodium pump ligand, marinobufagenin, via a protein kinase $\mathrm{C}$ dependent mechanism. Journal of Hypertension 18(2):209-215. DOI 10.1097/00004872200018020-00012

Baker AJ. 2014. Adrenergic signaling in heart failure: a balance of toxic and protective effects. Pflugers Archiv : European journal of physiology 466(6):1139-1150. DOI $10.1007 / \mathrm{s} 00424-014-1491-5$

Braz JC, Gregory K, Pathak A, Zhao W, Sahin B, Klevitsky R, Kimball TF, Lorenz JN, Nairn AC, Liggett SB, Bodi I, Wang S, Schwartz A, Lakatta EG, DePaoli-Roach AA, Robbins J, Hewett TE, Bibb JA, Westfall MV, Kranias EG, and Molkentin JD. 2004. $\mathrm{PKC}-\alpha$ regulates cardiac contractility and propensity toward heart failure. Nature Medicine 10(3):248-254. DOI 10.1038/nm1000

Chen JJ, Marsden AN, Scott CA, Akimzhanov AM, and Boehning D. 2020. DHHC5 Mediates $\beta$-Adrenergic Signaling in Cardiomyocytes by Targeting Ga Proteins. Biophysical Journal 118(4):826-835. https://doi.org/10.1016/j.bpj.2019.08.018

Chen S, Guo W, Qi X, Zhou J, Liu Z, and Cheng Y. 2019. Natural alkaloids from lotus plumule ameliorate lipopolysaccharide-induced depression-like behavior: integrating network pharmacology and molecular mechanism evaluation. Food \& Function 10(9):6062-6073. DOI 10.1039/c9fo01092k

Chinese Pharmacopoeia Commission. 2015. Pharmacopoeia of People's Republic of China 
Part I.. Beijing: People’s Medical Publishing House.

337

338

339

340

341

342

343

344

345

346

347

348

349

350

351

352

353

354

355

356

Cruz Jdos S, and Matsuda H. 1993. Arenobufagin, a compound in toad venom, blocks $\mathrm{Na}(+)-$

$\mathrm{K}+$ pump current in cardiac myocytes. European Journal of Pharmacology 239(1-3):223226. DOI 10.1016/0014-2999(93)90999-x

Dennis G, Jr., Sherman BT, Hosack DA, Yang J, Gao W, Lane HC, and Lempicki RA. 2003. DAVID: Database for Annotation, Visualization, and Integrated Discovery. Genome Biology 4(5):P3-P3.

Di Palo KE, and Barone NJ. 2020. Hypertension and Heart Failure: Prevention, Targets, and Treatment. Heart Failure Clinics 16(1):99-106. https://doi.org/10.1016/j.hfc.2019.09.001

Ding M, Ma W, Wang X, Chen S, Zou S, Wei J, Yang Y, Li J, Yang X, Wang H, Li Y, Wang Q, Mao H, Gao X, and Chang Y. 2019. A network pharmacology integrated pharmacokinetics strategy for uncovering pharmacological mechanism of compounds absorbed into the blood of Dan-Lou tablet on coronary heart disease. Journal of Ethnopharmacology 242:112055. https://doi.org/10.1016/j.jep.2019.112055

Dobrev D, and Wehrens XHT. 2010. Calmodulin Kinase II, Sarcoplasmic Reticulum Ca2+ Leak, and Atrial Fibrillation. Trends in Cardiovascular Medicine 20(1):30-34. https://doi.org/10.1016/j.tcm.2010.03.004

Fedorova OV, Talan MI, Agalakova NI, Droy-Lefaix MT, Lakatta EG, and Bagrov AY. 2003. Myocardial PKC beta 2 and the sensitivity of $\mathrm{Na} / \mathrm{K}-\mathrm{ATP}$ ase to marinobufagenin are reduced by cicletanine in Dahl hypertension. Hypertension 41(3):505-511. DOI 10.1161/01.Hyp.0000053446.43894.9f 
357

358

359

Griesenauer RH, Schillebeeckx C, and Kinch MS. 2019. Assessing the public landscape of clinical-stage pharmaceuticals through freely available online databases. Drug Discovery Today 24(4):1010-1016. DOI 10.1016/j.drudis.2019.01.010

Hamosh A, Scott AF, Amberger JS, Bocchini CA, and McKusick VA. 2005. Online Mendelian Inheritance in Man (OMIM), a knowledgebase of human genes and genetic disorders. Nucleic Acids Research 33:D514-D517. DOI 10.1093/nar/gki033

He R, Ma H, Zhou J, Zhu Z, Lv X, Li Q, Wang H, Yan Y, Luo N, Di L, Wu Q, and Duan J. 2019. High Resolution Mass Profile of Bufadienolides and Peptides Combing with AntiTumor Cell Screening and Multivariate Analysis for the Quality Evaluation of Bufonis Venenum. Molecules (Basel, Switzerland) 24(10):1943. DOI 10.3390/molecules24101943

He X, Hu H, Wu Y, and Zeng X. 2012. Urinary metabolites of cinobufagin in rats and their antiproliferative activities. Natural Product Research 26(6):489-499. DOI $10.1080 / 14786419.2010 .510798$

Jensen LJ, Kuhn M, Stark M, Chaffron S, Creevey C, Muller J, Doerks T, Julien P, Roth A, Simonovic M, Bork P, and von Mering C. 2009. STRING 8--a global view on proteins and their functional interactions in 630 organisms. Nucleic Acids Research 37:D412-D416. DOI 10.1093/nar/gkn760

Jia Q, Wang L, Zhang X, Ding Y, Li H, Yang Y, Zhang A, Li Y, Lv S, and Zhang J. 2020. Prevention and treatment of chronic heart failure through traditional Chinese medicine: Role of the gut microbiota. Pharmacological Research 151:104552. 
https://doi.org/10.1016/j.phrs.2019.104552

Lan Y, Wang X, Lou J-C, Xing J-S, Yu Z, Wang H, Zou S, Ma X, and Zhang B. 2018. Bufalin inhibits glioblastoma growth by promoting proteasomal degradation of the $\mathrm{Na}(+) / \mathrm{K}(+)$-ATPase $\alpha 1$ subunit. Biomedicine \& Pharmacotherapy 103:204-215. DOI 10.1016/j.biopha.2018.04.030

Laursen M, Gregersen JL, Yatime L, Nissen P, and Fedosova N. 2015. Structures and characterization of digoxin- and bufalin-bound $\mathrm{Na}+, \mathrm{K}+-\mathrm{ATPase}$ compared with the ouabain-bound complex. Proceedings of the National Academy of Sciences of the United States of America 112(6):1755-1760. DOI 10.1073/pnas. 1422997112

Liang Y, Liu A, Qin S, Sun J, Yang M, Li P, and Guo D. 2008. Simultaneous determination and pharmacokinetics of five bufadienolides in rat plasma after oral administration of Chansu extract by SPE-HPLC method. Journal of Pharmaceutical and Biomedical Analysis 46(3):442-448. https://doi.org/10.1016/j.jpba.2007.11.001

Lother A, and Hein L. 2016. Pharmacology of heart failure: From basic science to novel therapies. Pharmacology \& Therapeutics 166:136-149. https://doi.org/10.1016/j.pharmthera.2016.07.004

Luo Z, Yu G, Chen X, Liu Y, Zhou Y, Wang G, and Shi Y. 2020. Integrated phytochemical analysis based on UHPLC-LTQ-Orbitrap and network pharmacology approaches to explore the potential mechanism of Lycium ruthenicum Murr. for ameliorating Alzheimer's disease. Food \& Function 11(2):1362-1372. DOI 10.1039/c9fo02840d

Miao X, Zheng L, Zhao Z, Su S, Zhu, Guo J, Shang E, Qian D, and Duan J. 2019. 
Protective Effect and Mechanism of Boswellic Acid and Myrrha Sesquiterpenes with

Different Proportions of Compatibility on Neuroinflammation by LPS-Induced BV2

Cells Combined with Network Pharmacology. Molecules (Basel, Switzerland)

24(21):3946. DOI 10.3390/molecules24213946

403

Miyashiro Y, Nishio T, and Shimada K. 2008. Characterization of in vivo metabolites of toad venom using liquid chromatography-mass spectrometry. Journal of Chromatographic Science 46(6):534-538. DOI 10.1093/chromsci/46.6.534

Molkentin J, and Robbins J. 2009. With great power comes great responsibility: Using mouse genetics to study cardiac hypertrophy and failure. Journal of Molecular and Cellular Cardiology 46(2):130-136. https://doi.org/10.1016/j.yjmcc.2008.09.002

Moreno Y, Katz A, Miklos W, Cimmino A, Tal D, Ainbinder E, Zehl M, Urban E, Evidente A, Kopp B, Berger W, Feron O, Karlish S, and Kiss R. 2013. Hellebrin and its aglycone form hellebrigenin display similar in vitro growth inhibitory effects in cancer cells and binding profiles to the alpha subunits of the Na+/K+-ATPase. Molecular Cancer 12:33. DOI 10.1186/1476-4598-12-33.

Nguyen QK, Edward K, Nagasundaram N, Matthew C, and Yeh HY. 2019a. Computational identification of vesicular transport proteins from sequences using deep gated recurrent units architecture. Computational and Structural Biotechnology Journal 17:1245-1254. DOI 10.1016/j.csbj.2019.09.005.

Nguyen QK, Edward K, Nagasundaram N, Matthew C, and Yeh HY. 2019b. ET-GRU: using multi-layer gated recurrent units to identify electron transport proteins. BMC 
Bioinformatics 20:377. DOI: 10.1186/s12859-019-2972-5.

421 Ning J, Wu T, Tian Y, Wang C, Tian G, Zhang B, Liu K, and Ma X. 2010. Identification of cinobufagin metabolites in the bile of rats. Xenobiotica 40(1):48-54. DOI $10.3109 / 00498250903331049$

424

425

426

427

428

429

430

431

432

433

434

435

436

437

438

439

440

Orlov SN, Tverskoi AM, Sidorenko SV, Smolyaninova LV, Lopina OD, Dulin NO, and

Klimanova EA. 2020. Na,K-ATPase as a target for endogenous cardiotonic steroids: What's the evidence? Genes \& Diseases. https://doi.org/10.1016/j.gendis.2020.01.008

Pan Z, Luo Y, Xia Y, Zhang X, Qin Y, Liu W, Li M, Liu X, Zheng Q, and Li D. 2020. Cinobufagin induces cell cycle arrest at the $\mathrm{S}$ phase and promotes apoptosis in nasopharyngeal carcinoma cells. Biomedicine \& Pharmacotherapy 122:109763. https://doi.org/10.1016/j.biopha.2019.109763

Qin H, Chen H, Zou Y, Zhang X, Wei C, Chen W, Xie Z, Yao M, and Han B. 2019. Systematic investigation of the mechanism of Cichorium glandulosum on type 2 diabetes mellitus accompanied with non-alcoholic fatty liver rats. Food \& Function 10(5):24502460. DOI 10.1039/c8fo02284d

Santulli G, and Iaccarino G. 2016. Adrenergic signaling in heart failure and cardiovascular aging. Maturitas 93:65-72. https://doi.org/10.1016/j.maturitas.2016.03.022

Šeflová J, Čechová P, Biler M, Hradil P, and Kubala M. 2017. Inhibition of Na+/K+-ATPase by 5,6,7,8-tetrafluoro-3-hydroxy-2-phenylquinolin-4(1H)-one. Biochimie 138:56-61. https://doi.org/10.1016/j.biochi.2017.04.009

Shannon P, Markiel A, Ozier O, Baliga NS, Wang J, Ramage D, Amin N, Schwikowski B, 
441

442

443

444

445

446

447

448

449

450

451

452

453

454

455

456

457

458

459

460

461

and Ideker T. 2003. Cytoscape: a software environment for integrated models of biomolecular interaction networks. Genome Research 13(11):2498-2504. DOI

$10.1101 /$ gr.1239303

Sousa LQ, Machado KD, Oliveira SF, Araújo LD, Monção-Filho ED, Melo-Cavalcante AA, Vieira-Júnior GM, and Ferreira PM. 2017. Bufadienolides from amphibians: A promising source of anticancer prototypes for radical innovation, apoptosis triggering and $\mathrm{Na}(+) / \mathrm{K}(+)$-ATPase inhibition. Toxicon 127:63-76. DOI 10.1016/j.toxicon.2017.01.004

Strauss M, Smith W, Fedorova OV, and Schutte AE. 2019. The $\mathrm{Na}(+) \mathrm{K}(+)-A T P a s e$ Inhibitor Marinobufagenin and Early Cardiovascular Risk in Humans: a Review of Recent Evidence. Current Hypertension Reports 21(5):38. DOI 10.1007/s11906-019-0942-y

Tao J, Mao L, Zhou B, Liu Q, Yang A, Wei G, Liu R, Zhang W, Xu W, and Ye J. 2017. Simultaneous determination of ginsenosides and bufadienolides in rat plasma after the oral administration of Shexiang Baoxin Pill for pharmacokinetic study by liquid chromatography tandem mass spectrometry following solid-phase extraction. Biomedical chromatography 31(3). DOI 10.1002/bmc.3816

Wang Z, Sun L, and Heinbockel T. 2014. Resibufogenin and cinobufagin activate central neurons through an ouabain-like action. Plos One 9(11):e113272. DOI 10.1371/journal.pone.0113272

Wei W, An Y, Zhang Y, Li Z, Zhou Y, Lei M, Zhang J, Qu H, Da J, Wu W, and Guo D. 2020. Quantitative analysis of fourteen bufadienolides in Venenum Bufonis crude drug and its Chinese patent medicines by ultra-high performance liquid chromatography 
coupled with tandem mass spectrometry. Journal of Ethnopharmacology 251:112490. https://doi.org/10.1016/j.jep.2019.112490

Wei W, Hou J, Wang X, Yu Y, Li H, Li Z, Feng Z, Qu H, Wu W, and Guo D. 2019.

Venenum bufonis: An overview of its traditional use, natural product chemistry, pharmacology, pharmacokinetics and toxicology. Journal of Ethnopharmacology 237:215-235. https://doi.org/10.1016/j.jep.2019.03.042

Wu S, Hsiao Y, Kuo C, Yu F, Hsu S, Wu P, Chen J, Hsia T, Liu H, Hsu W, and Chung J. 2015. Bufalin Inhibits NCI-H460 Human Lung Cancer Cell Metastasis In Vitro by Inhibiting MAPKs, MMPs, and NF-kB Pathways. The American journal of Chinese medicine 43(6): 1247-1264. DOI 10.1142/s0192415x15500718

Xia X, Jin H, Yan S, and Zhang W. 2010. Analysis of the bioactive constituents of ChanSu in rat plasma by high performance liquid chromatography with mass spectrometric detection. Journal of Pharmaceutical and Biomedical Analysis 53(3):646-654. DOI 10.1016/j.jpba.2010.05.009

Xin X, Dong P, Sun X, Deng S, Zhang N, Wang C, Huo X, Li Y, Lan R, Chen L, and Fan G. 2016. Identification of the hydroxylated derivatives of bufalin: phase I metabolites in rats. Journal of Asian Natural Products Research 18(3):239-247. DOI $10.1080 / 10286020.2015 .1071358$

Xu S, Wang Y, Yu M, Wang D, Liang Y, Chen Y, Liao C, Xie Z, Zhao B, Han J, Duan Y, and Yang X. 2020. LongShengZhi capsule inhibits doxorubicin-induced heart failure by anti-oxidative stress. Biomedicine \& Pharmacotherapy 123:109803. 
483

484

485

486

487

488

489

490

491

492

493

494

495

496

497

498

499

500

501

502

503

https://doi.org/10.1016/j.biopha.2019.109803

Yang L, Li A, Chen M, Yan Y, Liu Y, Li K, Jia J, and Qin X. 2020. Comprehensive investigation of mechanism and effective ingredients of Fangji Huangqi Tang by serum pharmacochemistry and network pharmacology. Biomedical chromatography 34(4):e4785. DOI 10.1002/bmc.4785

Yang Y, Shi C, Xie J, Dai J, He S, Tian Y. 2020. Identification of Potential Dipeptidyl Peptidase (DPP)-IV Inhibitors among Moringa oleifera Phytochemicals by Virtual Screening, Molecular Docking Analysis, ADME/T-Based Prediction, and In Vitro Analyses. Molecules 25(1):189. DOI: 10.3390/molecules25010189.

Yu G, Luo Z, Zhou Y, Zhang L, Wu Y, Ding L, and Shi Y. 2019. Uncovering the pharmacological mechanism of Carthamus tinctorius L. on cardiovascular disease by a systems pharmacology approach. Biomedicine \& Pharmacotherapy 117:109094. https://doi.org/10.1016/j.biopha.2019.109094

Yu G, Wang W, Wang X, Xu M, Zhang L, Ding L, Guo R, and Shi Y. 2018. Network pharmacology-based strategy to investigate pharmacological mechanisms of Zuojinwan for treatment of gastritis. BMC Complementary and Alternative Medicine 18(1):292-292. DOI 10.1186/s12906-018-2356-9

Yu G, Zhang Y, Ren W, Dong L, Li J, Geng Y, Zhang Y, Li D, Xu H, and Yang H. 2016. Network pharmacology-based identification of key pharmacological pathways of YinHuang-Qing-Fei capsule acting on chronic bronchitis. International Journal of Chronic Obstructive Pulmonary Disease 12:85-94. DOI 10.2147/COPD.S121079 
504

505

506

507

508

509

510

511

512

513

514

515

516

517

518

519

520

521

522

523

524

Yun HR, Yoo HS, Shin DY, Hong SH, Kim J-H, Cho CK, and Choi YH. 2009. Apoptosis Induction of Human Lung Carcinoma Cells by Chan Su (Venenum Bufonis) Through Activation of Caspases. Journal of Acupuncture and Meridian Studies 2(3):210-217. https://doi.org/10.1016/S2005-2901(09)60057-1

Zhang X, Pi Z, Zheng Z, Liu Z, and Song F. 2018. Comprehensive investigation of in-vivo ingredients and action mechanism of iridoid extract from Gardeniae Fructus by liquid chromatography combined with mass spectrometry, microdialysis sampling and network pharmacology. Journal of Chromatography B 1076:70-76.

https://doi.org/10.1016/j.jchromb.2018.01.023

Zhang Z, Yi P, Yang J, Huang J, Xu P, Hu M, Zhang C, Wang B, and Peng W. 2020. Integrated network pharmacology analysis and serum metabolomics to reveal the cognitive improvement effect of Bushen Tiansui formula on Alzheimer's disease. Journal of Ethnopharmacology 249:112371. https://doi.org/10.1016/j.jep.2019.112371

Zhu Z, Deng S, Liu D, Zhang BJ, Sun HZ, Tian Y, Wang CY, Wang L, and Ma X. 2013. Isolation and identification of phase I metabolites of resibufogenin in rats. Xenobiotica 43(5):479-485. DOI 10.3109/00498254.2012.728728

\section{Figure Captions}

Fig. 1. Workflow of network pharmacology and molecular docking approaches to reveal the active components and molecular mechanisms of VB acting on HF.

Fig. 2. GO term performance and pathway enrichment analysis of the major hubs. (a) GO: BP; 
(b) GO: MF; (c) GO: CC; and (d) KEGG. The ordinate stands for GO terms or the main

526

527

528

529

530

531

532

533

534

535

536

537

538

539

540

541

542

543

544

545

pathways, the primary abscissa stands for minus $\log 10(P)$, and the secondary abscissa stands for the percentage of major hubs involved in the corresponding GO terms or the main pathways out of total major hubs.

Fig. 3. VB ingredients-major hubs-pathway network.

Green V-diagrams represent each prototype component in VB; yellow V-diagrams represent each metabolite in VB; round blue nodes represent putative targets of components in VB; round red nodes represent known therapeutic targets for HF; orange rectangles represent top 11 pathways from enrichment analysis of major targets; edges represent interactions among VB ingredients, putative targets, known therapeutic targets for the treatment of $\mathrm{VB}$, and main pathways.

Fig. 4. Adrenergic signalling in cardio-myocytes influenced by major putative targets of VB components.

Fig. 5. The binding modes of the selected compounds and targets.

(a) Schematic (3D) representation and (b) Schematic (2D) representation of the interplay between bufotalin and ATP1A1 (PDB IDchimeric 3N23).

(c) Schematic (3D) representation and (d) Schematic (2D) representation of the interplay between cinobufaginol and GNAS (PDB IDchimeric 3C14).

(e) Schematic (3D) representation and (f) Schematic (2D) representation of the interplay between cinobufaginol and MAPK1 (PDB IDchimeric 3071).

(g) Schematic (3D) representation and (h) Schematic (2D) representation of the interplay 
546 between 19-oxo-bufalin and PRKCA (PDB IDchimeric 4DNL).

547 Active site amino acid residues were represented as tubes, while the compounds were shown as 548 stick model with purple colored. 
Table $\mathbf{1}$ (on next page)

Molecular docking results (Top 5 for each targets) 


\section{$1 \quad$ Table 1}

2 Molecular docking results (Top 5 for each targets).

\begin{tabular}{|c|c|c|}
\hline Targets & Compound & -CIE (kcal/mol) \\
\hline \multirow[t]{5}{*}{ ATP1A1 } & Bufotalin & 49.0335 \\
\hline & Cinobufotalin & 46.109 \\
\hline & Cinobufagin & 45.1269 \\
\hline & Cinobufaginol & 44.0494 \\
\hline & $5 \beta, 6 \alpha$-dihydroxybufalin & 43.3908 \\
\hline \multirow[t]{5}{*}{ GNAS } & Cinobufaginol & 55.2668 \\
\hline & 6a-hydroxybufalin & 53.1182 \\
\hline & 19-oxo-desacetylcinobufagin & 52.2151 \\
\hline & 1,5-dihydroxyldesacetylcinobufagin & 49.9538 \\
\hline & $1,12 \beta$-dihydroxycinobufagin & 49.4652 \\
\hline \multirow[t]{5}{*}{ MAPK1 } & Cinobufaginol & 55.1432 \\
\hline & Cinobufagin & 54.0654 \\
\hline & Bufotalin & 53.1891 \\
\hline & 1,5-dihydroxyldesacetylcinobufagin & 51.5985 \\
\hline & 12-hydroxyl-cinobufagin & 51.2183 \\
\hline \multirow[t]{2}{*}{ PRKCA } & 19-oxo-bufalin & 42.0454 \\
\hline & Marinobufagin & 39.3428 \\
\hline
\end{tabular}


Hellebrigenin

$5 \beta, 6 \alpha$-dihydroxybufalin

Resibufogenin
38.6171

37.9654

37.8932

3 
Figure 1

Workflow of network pharmacology and molecular docking approaches to reveal the active components and molecular mechanisms of VB acting on $\mathrm{HF}$ 


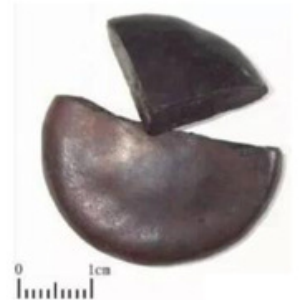

Venenum Bufonis (VB)

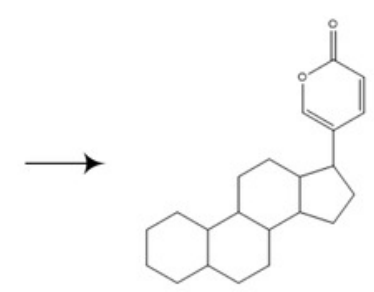

In vivo constituents of $\mathrm{VB}$

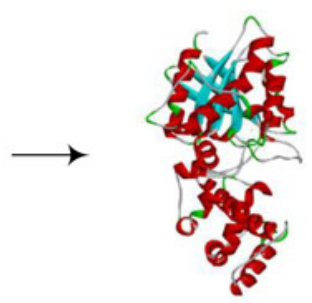

Targets of VB

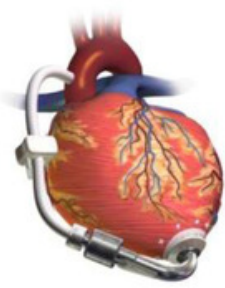

Heart Failure

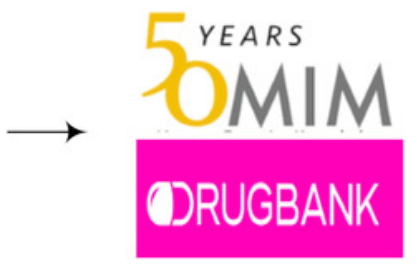

Disease associated databases

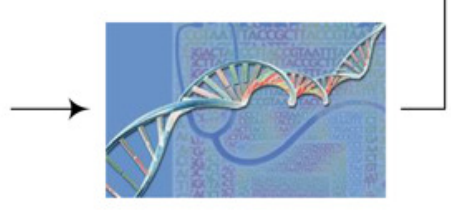

Disease associated genes

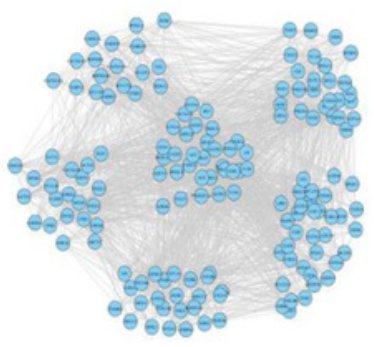

Network analysis

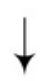

KEGG

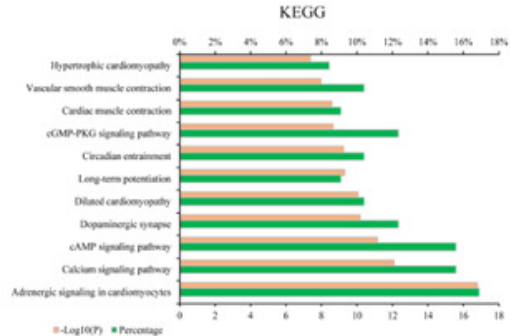

GO\&KEGG analysis

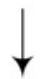

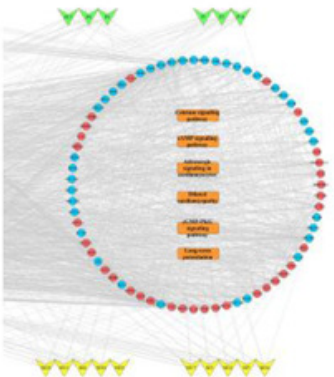

Chemical-TargetPathway network

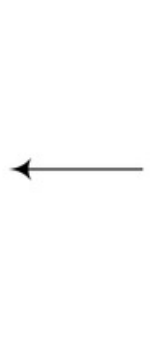

$\forall$ 
Figure 2

GO term performance and pathway enrichment analysis of the major hubs.

(a) GO: BP; (b) GO: MF; (c) GO: CC; and (d) KEGG. The ordinate stands for GO terms or the main pathways, the primary abscissa stands for minus log $10(P)$, and the secondary abscissa stands for the percentage of major hubs involved in the corresponding GO terms or the main pathways out of total major hubs.
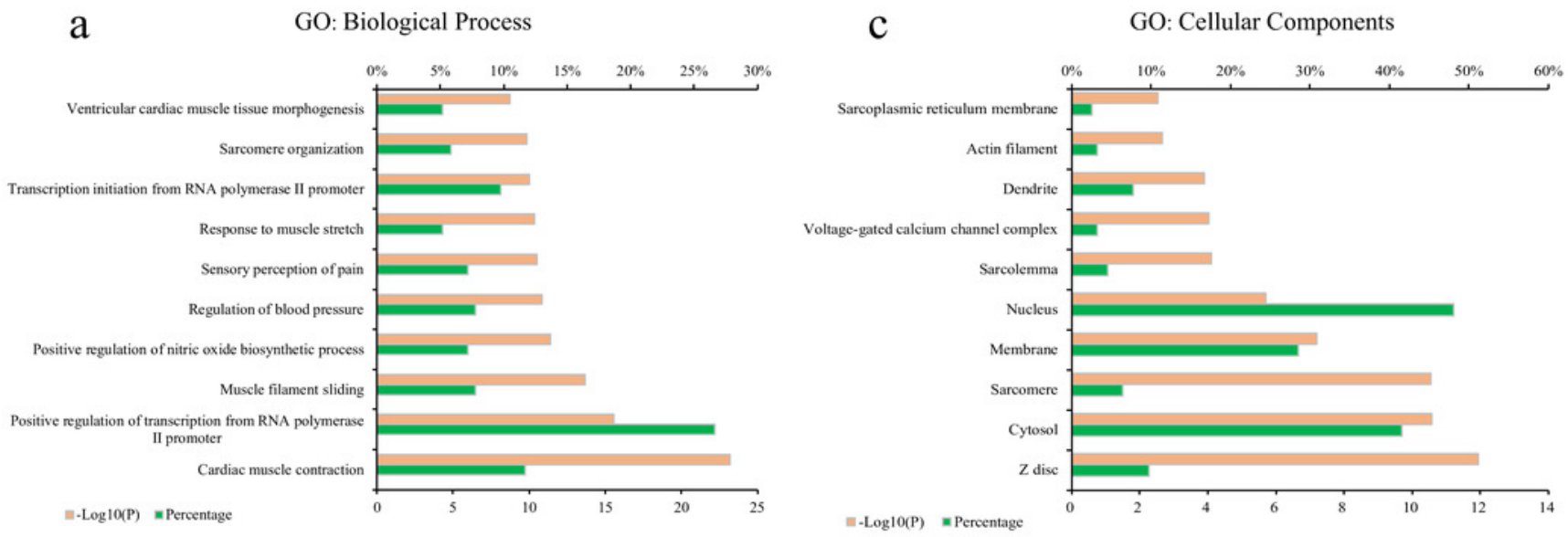

b

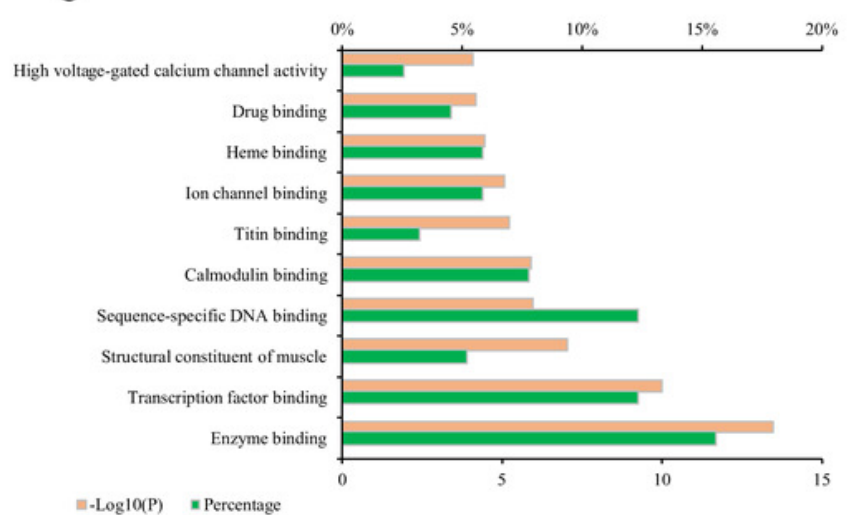

$\mathrm{d}$

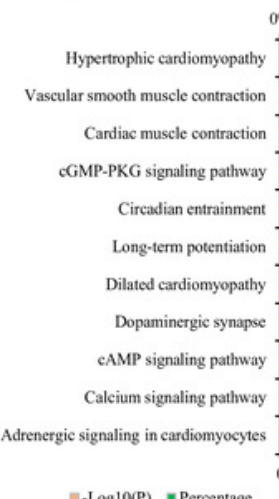

KEGG Pathways

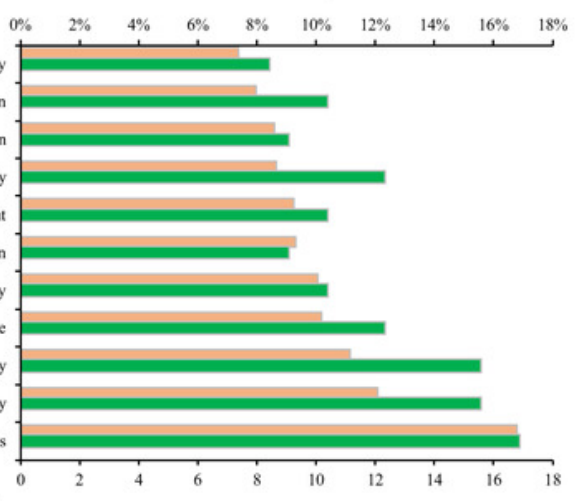




\section{Figure 3}

VB ingredients-major hubs-pathway network.

Green V-diagrams represent each prototype component in VB; yellow V-diagrams represent each metabolite in VB; round blue nodes represent putative targets of components in VB; round red nodes represent known therapeutic targets for HF; orange rectangles represent top 11 pathways from enrichment analysis of major targets; edges represent interactions among VB ingredients, putative targets, known therapeutic targets for the treatment of VB, and main pathways. 


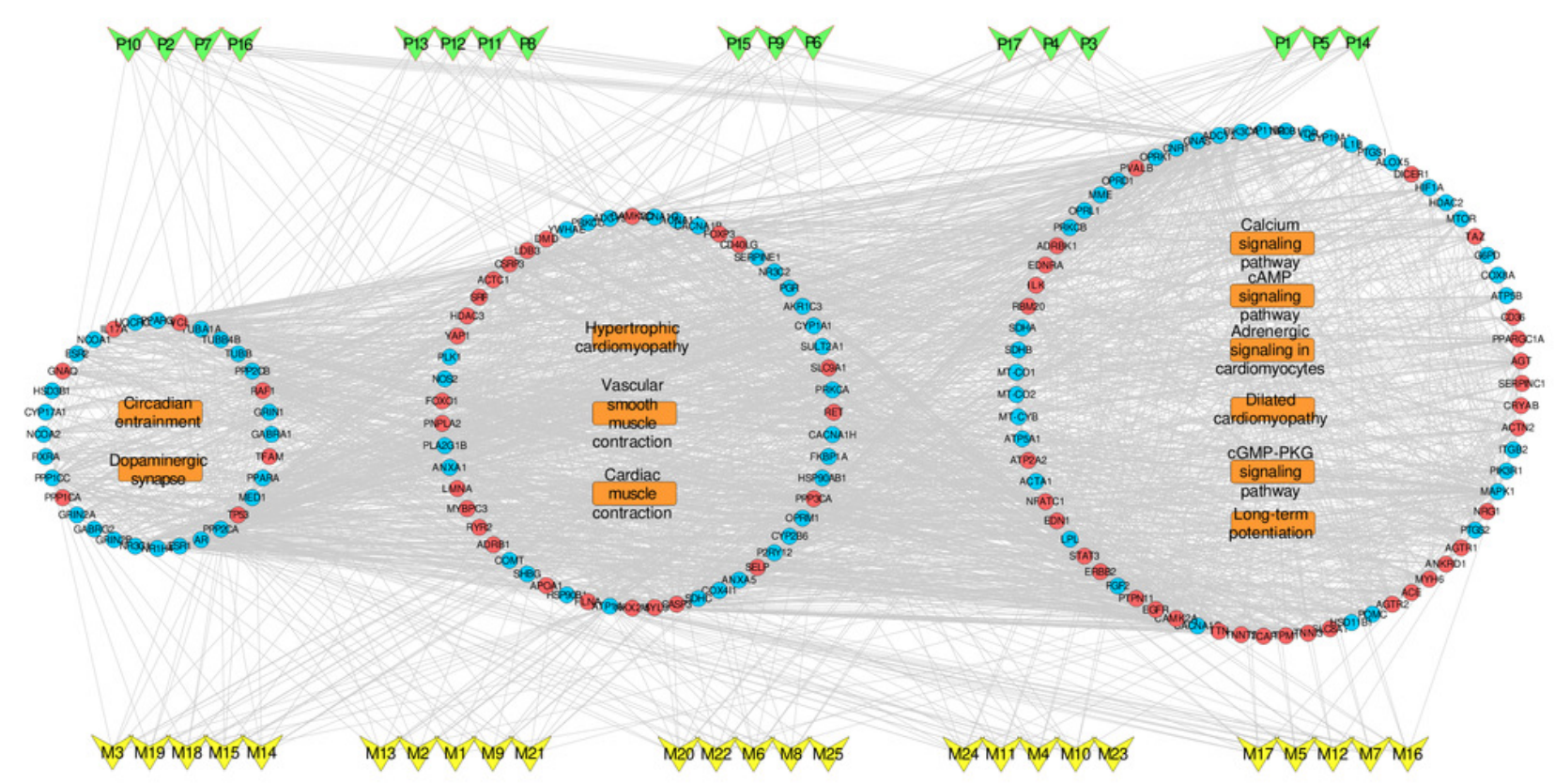


Figure 4

Adrenergic signalling in cardio-myocytes influenced by major putative targets of VB components.

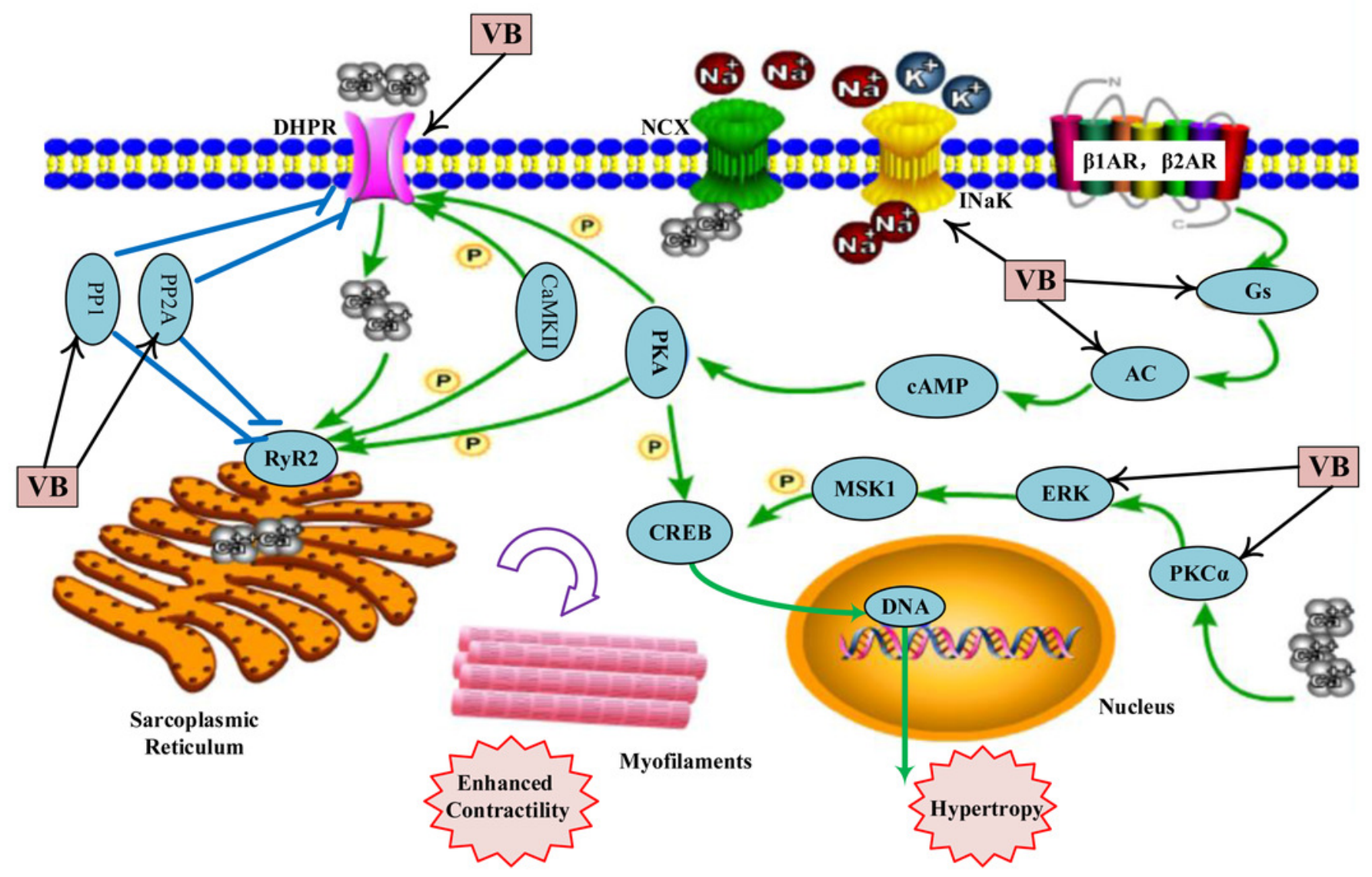




\section{Figure 5}

The binding modes of the selected compounds and targets.

(a) Schematic (3D) representation and (b) Schematic (2D) representation of the interplay between bufotalin and ATP1A1 (PDB IDchimeric 3N23). (c) Schematic (3D) representation and (d) Schematic (2D) representation of the interplay between cinobufaginol and GNAS (PDB IDchimeric 3C14). (e) Schematic (3D) representation and (f) Schematic (2D) representation of the interplay between cinobufaginol and MAPK1 (PDB IDchimeric 3071). (g) Schematic (3D) representation and (h) Schematic (2D) representation of the interplay between 19-oxobufalin and PRKCA (PDB IDchimeric 4DNL). Active site amino acid residues were represented as tubes, while the compounds were shown as stick model with purple colored. 


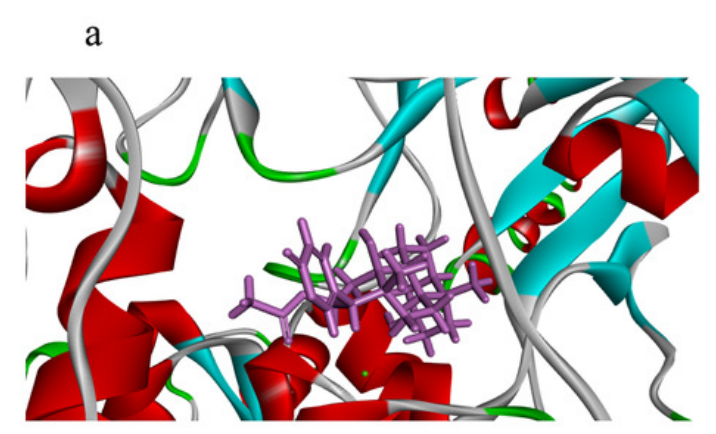

b

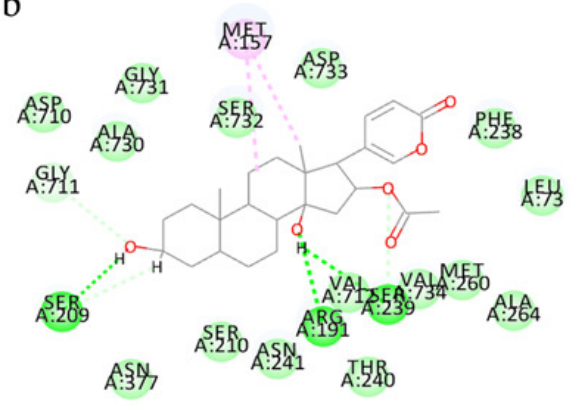

Interactions

$\square$ van der Waals
Conventional Hydrogen Bond
$\square$ Carbon Hydrogen Bond

e

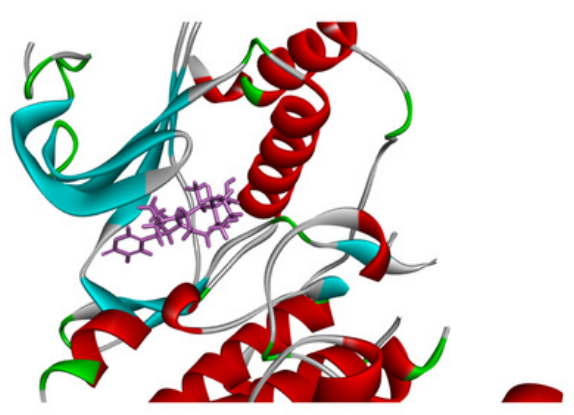

$\mathrm{f}$

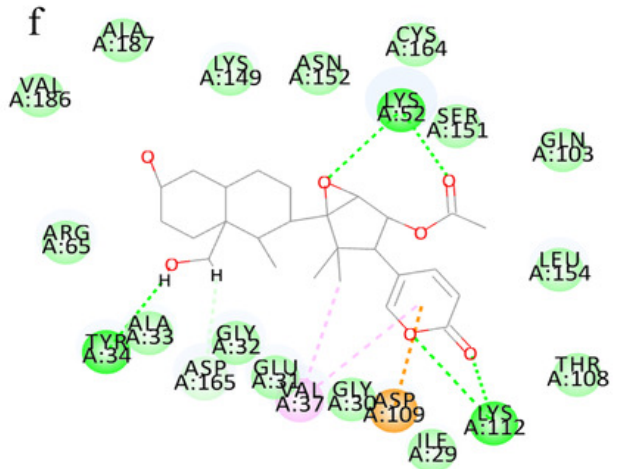

Interactions

$\square$ van der Waals

Conventional Hydrogen Bond

$\square$ Carbon Hydrogen Bond

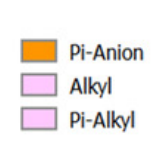

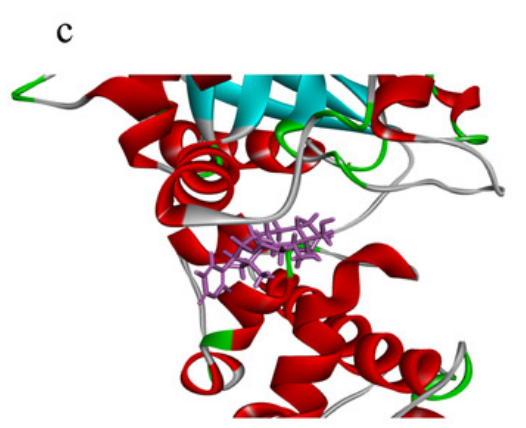

d
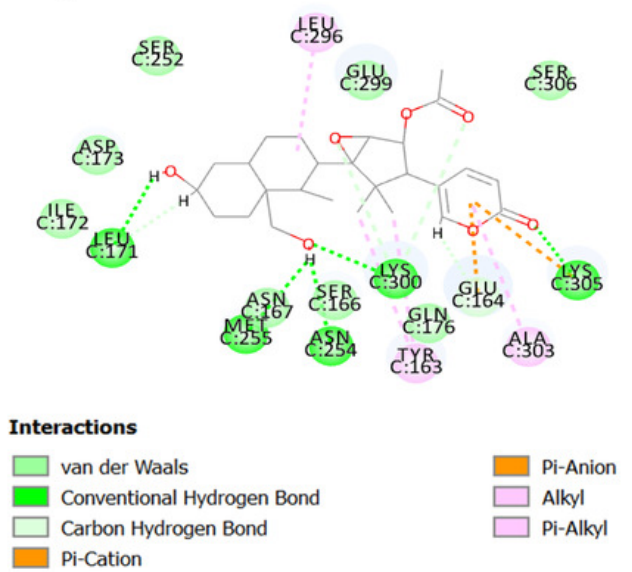

g

$\mathrm{h}$
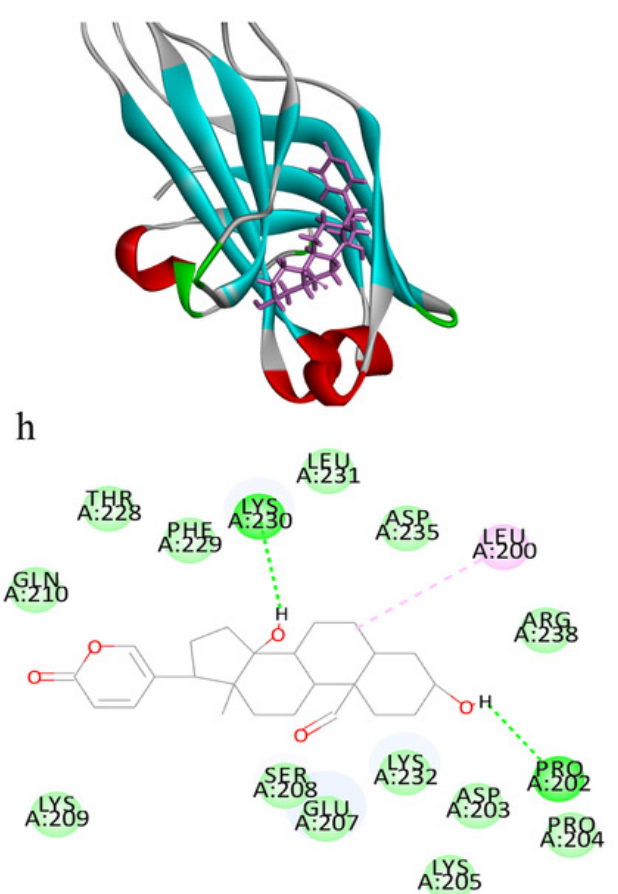

Interactions

van der Waals

Conventional Hydrogen Bond 